\title{
Patrones de participación y logro en un MOOC de Saberes Digitales para Docentes en servicio de México
}

\author{
Participation Patterns and Achievement in a MOOC on Digital Knowledge for Mexican \\ In-Service Teacher Training
}

Patrons de participació i assoliment en un MOOC de Sabers Digitals per a Docents de Mèxic

\author{
Ricardo Mercado-del-Collado*(๑), Nancy Jácome-Avila ${ }^{\circledR}$, Juan Carlos \\ Ortega-Guerrero $^{\circledR}$, Miguel Ángel Casillas-Alvarado ${ }^{\circledR}$, Alberto Ramírez-Martinell ${ }^{\circledR}$ \\ Centro de Investigación e Innovación en Educación Superior, Universidad Veracruzana, Xalapa, Veracruz, \\ México \\ ${ }^{*}$ Autor para correspondencia.
}

Recibido: 10/10/2019 | Aceptado: 09/12/2019 | Publicado: 24/12/2019

Cómo citar:
Mercado-del-Collado, R.;
Jácome-Avila, N.;
Ortega-Guerrero, J. C.;
Casillas-Alvarado, M. A.; y
Ramírez-Martinell, A.
(2019). Patrones de
participación y logro en un
MO0C de Saberes Digitales
para Docentes en servicio
de México. Research in
Education and Learning
Innovation Archives,
23,80-96.
10.7203/realia.23.15904

Copyright: El/La Autor/a. Open Access: Este es un artículo de acceso abierto distribuido bajo los términos de la licencia Creative Commons AttributionNonCommercial-ShareAlike 4.0 International (CC BY-NC-SA 4.0)

Financiación: None informed
RESUMEN: En este artículo se investigaron los patrones de participación y logro de más de 8000 participantes en un MOOC de Saberes Digitales para Docentes mediante estadística descriptiva y análisis de conglomerados en función de edad, género y nivel educativo. Los resultados indican que los participantes de mayor edad y nivel educativo obtuvieron mejores niveles de logro y participaron más en el foro de discusión. No se encontraron diferencias significativas entre género. La participación global siguió un modelo de embudo en donde existe un decremento notable de la participación a partir de la primera actividad para estabilizarse a partir de la quinta. El curso descansó sobre una base de confianza en la que se asume al participante como par académico que ha optado por tomar voluntariamente el curso con el propósito principal de aprender. Por lo que considerar la autopercepción de su desempeño como parte de la guía de avance es consistente con la filosofía de estos cursos.

PALABRAS CLAVE: MOOCs; formación de docentes en servicio; cursos masivos abiertos en línea

ABSTRACT: Participation patterns and completion rates of a MOOC on Digital Knowledge for Teachers with over 8000 students were investigated in this article using descriptive statistics and cluster analysis according to age, gender and previous educational attainment. Results indicate that older and more educated participants obtained higher completion rates and employed more often the discussion forum. No gender differences were obtained. Participation in the course followed a funnel model in which there is a steep decrease of involvement after the first activity which stabilizes in the fifth one. The course rested on a basis of trust assuming that each participant voluntarily enrolled with the purpose of learning and therefore considering his/her self-perception of course performance as one element of overall appraisal is consistent with these courses.

KEYWORDS: MOOCs; in-service training; massive online open courses

RESUM: En aquest article es van investigar els patrons de participació i assoliment de més de 8000 participants en un MOOC de Sabers Digitals per a Docents mitjançant estadística descriptiva i anàlisi de conglomerats en funció d'edat, gènere i nivell educatiu. Els resultats indiquen que els participants 
de major edat i nivell educatiu van obtenir millors nivells d'assoliment i van participar més en el fòrum de discussió. No es van trobar diferències significatives entre gèneres. La participació global va seguir un model d'embut on hi ha un decrement notable de la participació a partir de la primera activitat per estabilitzar-se a partir de la quinta. Es discuteix sobre la responsabilitat que han de demostrar els mestres en els programes de formació en servei. El curs va descansar sobre una base de confiança en la cual s'assumeix al participant con a parell acadèmic que ha optat per prendre voluntàriament el curs amb el propòsit principal d'aprendre. Per això considerar l'autopercepció del seu acompliment com a part de la guia d'avanc és consistent amb la filosofia d'aquests cursos.

PARAULES CLAUC: MOOCs; formació de docents en servei; cursos massius oberts en línia

\section{Notas de aplicación práctica}

\section{Qué se sabe sobre este tema}

- El estudio sobre la relación entre variables demográficas como edad, género y nivel educativo y los patrones de participación y logro de los participantes en los cursos MOOC se ha llevado a cabo principalmente en los países desarrollados. Se conoce que sí existe una relación entre dichas variables y la participación y logro de los participantes.

\section{Qué aporta este trabajo}

- Es un primer acercamiento para conocer cómo participan y qué logros obtienen los docentes en servicio de México en un curso MOOC sobre saberes digitales.

- Se encontró que los resultados de participación y logro corresponden a lo encontrado en estudios previos realizados en países diferentes a México.

- Las personas de mayor edad y mayor nivel educativo presentan mejores tasas de participación y logro en un curso MOOC.

- En este estudio se consideró a la autoevaluación de los participantes como un elemento adicional en la valoración de su desempeño, apelando al sentido de responsabilidad asumido por los docentes que aceptan participar voluntariamente en esta modalidad.

\section{Implicaciones para la práctica y/o política}

- Con base en los resultados obtenidos en este estudio se considera que los MOOC constituyen una estrategia eficaz para la formación continua de los docentes en servicio.

- Es importante considerar nuevas formas de evaluar la eficiencia de los cursos MOOC pues las poblaciones atendidas poseen motivaciones diversas para participar.

\section{INTRODUCCIÓN}

Los cursos masivos abiertos en línea (MOOC por sus siglas en inglés) son una tendencia mundial con más de 100 millones de participantes. Hay más de 900 instituciones alrededor del mundo involucradas en la producción de una cifra superior a los $11 \mathrm{mil}$ cursos en esta modalidad (Shah, 2018). El origen de estos cursos se remonta al año 2008 con el curso ofrecido por la Universidad de Manitoba en Canadá por Stephen Downes y George Siemens con 25 estudiantes inscritos en la universidad y más de 2000 participantes externos a la institución que ingresaron de forma gratuita (Downes, 2012). En los once años desde su aparición esta modalidad ha crecido de manera notable.

Un sector que se ha beneficiado del potencial que representan los MOOC es el de la formación y capacitación de profesores en servicio. Misra (2018) refiere las múltiples iniciativas desarrolladas en esta área por instituciones como el Consejo Británico, la Universidad de California, la Universidad Estatal de Arizona, la Universidad de Groningen, la Universidad de Auckland y la Universidad Autónoma de Barcelona, entre muchas otras. Asimismo, hace un recuento de las iniciativas gubernamentales de formación de maestros puestas en marcha en países como Malasia, Tailandia, China, India y Corea del Sur para aprovechar el potencial que representan los MOOCs para 
apoyar la formación y capacitación permanente de sus maestros. Por su parte, Dikke y Faltin (2015) refieren que solo en el buscador MOOC List se encuentran más de 130 cursos dirigidos a la formación y capacitación de los docentes. Estos cursos se ofrecen en diferentes plataformas como Coursera, Canvas Network, FutureLearn, Miríada X, FUN, MOOC-Ed, Novo Ed y edX. El uso de MOOCs en la formación y capacitación de profesores en servicio constituye un área de interés creciente entre las comunidades académicas (Cassany y Lenguaje y Textos, 2014; Gulbahar, Kalelioglu, y Bahadir-Kert, 2018; Kaul, Aksela, y Wu, 2018; Kumari, 2016; Laurillard, 2016; León-Urrutia, Fielding, y White, 2016; Patru y Balaji, 2016; Silva-Peña y Salgado, 2014).

Es indudable que esta modalidad reúne condiciones que favorecen la creación de mayores oportunidades de aprendizaje y lo hacen especialmente para los maestros que diariamente están frente a grupo y disponen de poco tiempo para participar en programas de formación y capacitación que requieren su presencia en lugares y tiempos determinados. En cambio, los MOOCs son flexibles dado que usualmente son asíncronos y no requieren que los participantes estén en un mismo lugar y a una misma hora (Baturay, 2015); pueden ser abiertos y gratuitos lo cual apoya la inclusión y reduce brechas entre la población objetivo (Dellarocas y Alstybne, 2013); asimismo, son escalables por lo que alcanzan poblaciones masivas (Drake, Hara, y Seeman, 2015) y constituyen una oferta educativa con una relación costo-beneficio favorable (Florentine, 2015).

En México la producción de cursos MOOC es relativamente reciente (Mercado-del Collado et al., 2018; Ramírez-Montoya y Farías-Gaytán, 2018; Zubieta y Rama, 2015), si bien la población sumada de los cursos ofrecidos por la UNAM, el Tec de Monterrey y los ofrecidos mediante la plataforma de MéxicoX ya supera los seis millones de personas (Mercado-del Collado et al., 2018), son pocos los estudios reportados sobre su uso en la formación y capacitación de profesores en servicio. El primer MOOC latinoamericano fue dirigido a la población docente bajo la responsabilidad del Tec de Monterrey en 2013 mediante su participación en la Comunidad Latinoamericana Abierta Regional de Investigación Social y Educativa (CLARISE); el curso MOOC fue diseñado con el fin de promover el uso de recursos educativos abiertos entre los docentes de América Latina (Ramírez-Montoya y Farías-Gaytán, 2018). Más recientemente, García, Ibarra, y Sandoval (2018) reportan la impartición de dos MOOC dirigidos a los docentes del sistema del Colegio Nacional de Educación Profesional Técnica (CONALEP).

Es alentador conocer el desarrollo de esta modalidad en México, pero desafortunadamente es poco lo que sabemos acerca de los resultados alcanzados de los MOOC en términos de eficiencia terminal, trayectorias de participación y aprendizajes logrados, entre otros factores. Una razón de lo anterior es que no existe suficiente documentación publicada acerca de las experiencias de las instituciones participantes y de los cursos ofrecidos (Mercado-del Collado et al., 2018).

En particular en este trabajo interesa investigar la medida en que estos cursos representan una estrategia útil para la capacitación de los maestros en servicio de México y conocer los patrones de su participación y nivel de logro en función de sus características demográficas. Este tema ha sido investigado poco en México (Domínguez y Morcillo, 2016), no así en otros países en donde la relación entre variables demográficas y patrones de participación y logro en cursos MOOC se ha investigado ampliamente (Coffrin, Corrin, de Barba, y Kennedy, 2014; Phan, McNeil, y Robin, 2016).

En México las necesidades de formación y actualización de los docentes involucran a más de un millón de personas, distribuidas por todo el territorio nacional. En el contexto de la revolución tecnológica y de la cultura digital es imperiosa e indispensable una estrategia de actualización para el magisterio que garantice su inclusión a la sociedad digital con un alto dominio tecnológico y favorezca el cambio y la modernización en sus prácticas de enseñanza. Para contribuir en esta dirección, Ramírez y Casillas desarrollaron un MOOC con contenidos básicos que ha estado operando en 
la plataforma México X y que en tres ediciones ha tenido buena aceptación (RamírezMartinell y Casillas, 2015; Ramírez-Martinell, Casillas, y Aguirre, 2018; Veracruzana), 2019).

\section{HABILITACIÓN TECNOLÓGICA DE DOCENTES}

El MOOC Saberes Digitales para Docentes Nivel 1 surge como resultado de investigaciones que se llevaron a cabo en el Centro de Investigación e Innovación en Educación Superior (CIIES) de la Universidad Veracruzana (UV). El tema de saberes digitales (Ramírez-Martinell y Casillas, 2015) ha sido central en una línea de investigación procurada en el CIIES. El concepto se aplicó tanto en el contexto de la educación superior como de la educación básica, siendo este último una oportunidad para proponer el perfil tecnológico de los docentes de ese nivel educativo (Ramírez-Martinell y Casillas, 2017). Con la imagen tecnológica deseable de los docentes se procedió a diseñar un programa de capacitación encaminado a habilitarlos tecnológicamente.

Como antecedentes adicionales del MOOC se desarrollaron en el CIIES dos diplomados de Saberes Digitales para profesionales de la educación. Uno para docentes de educación básica y otro para profesores universitarios (Ramírez-Martinell et al., 2018).

De este modo, el MOOC Saberes Digitales para Docentes nivel 1 es el resultado de investigación, experiencia en el campo e innovación educativa y busca atender a un sector específico de la sociedad: los docentes en activo de la educación básica.

El MOOC de Saberes Digitales para docentes nivel 1, es el primer componente de un programa de formación docente compuesto por tres niveles. Desde su lanzamiento en octubre de 2018, el nivel 1 se ha ofrecido en tres ocasiones convocando a cerca de 20 mil interesados en el tema. La noción de "saberes digitales se refiere a una estructura graduada de conocimientos y habilidades tecnológicas que hacen observables las nociones y destrezas que poseen los actores educativos frente a las Tecnologías de la Información y de la Comunicación (TIC)" (Ramírez-Martinell y Casillas, 2018, p.10). Los diez saberes digitales son:

Manejo de sistemas digitales

- Saber usar dispositivos

- Saber administrar archivos

- Saber usar programas y sistemas de información especializados

Manipulación de contenido digital

- Saber crear y manipular contenido de texto y texto enriquecido

- Saber crear y manipular conjuntos de datos

- Saber crear y manipular medios y multimedia

Comunicación y socialización en entornos digitales

- Saber comunicarse en entornos digitales

- Saber socializar y colaborar en entornos digitales

Manejo de Información

- Saber ejercer y respetar una ciudadanía digital

- Literacidad digital 
En la siguiente tabla (Tabla 1 ) se detalla la dosificación de saberes para el nivel 1 (Ramírez-Martinell y Casillas, 2018).

Tabla 1. Dosificación de contenido del Nivel 1 del MOOC Saberes digitales para docentes

\begin{tabular}{|c|c|}
\hline Saber digital & Nivel 1 \\
\hline Saber administrar dispositivos digitales & $\begin{array}{l}\text { Manejar los recursos nativos de un teléfono } \\
\text { inteligente -fotos, video, audio, texto y comunicación- }\end{array}$ \\
\hline Saber administrar archivos informáticos & $\begin{array}{l}\text { Manejar funciones operativas para un uso básico de } \\
\text { archivos: reconocer archivos, ordenar carpetas, } \\
\text { comprimir y explorar sus propiedades }\end{array}$ \\
\hline $\begin{array}{l}\text { Saber usar programas y sistemas de } \\
\text { información especializados }\end{array}$ & Realizar presentaciones digitales \\
\hline Literacidad digital & Utilizar buscadores de información \\
\hline $\begin{array}{l}\text { Saber crear y manipular texto y texto } \\
\text { enriquecido }\end{array}$ & $\begin{array}{l}\text { Elaborar documentos de texto con estilo (tipo de } \\
\text { letra, cursivas, negritas, color, tamaño) y formato } \\
\text { (párrafo, interlineado) }\end{array}$ \\
\hline Saber crear y manipular conjuntos de datos & $\begin{array}{l}\text { Usar calculadora preinstalada en el teléfono } \\
\text { inteligente y una funcionalidad similar en la web }\end{array}$ \\
\hline Saber comunicarse en entornos digitales & $\begin{array}{l}\text { Comunicarse con recursos nativos del teléfono } \\
\text { inteligente como teléfono, mensajes y correo } \\
\text { electrónico }\end{array}$ \\
\hline $\begin{array}{l}\text { Saber socializar y colaborar en entornos } \\
\text { digitales }\end{array}$ & Utilizar grupos de colaboración en redes sociales \\
\hline Saber ejercer y respetar una ciudadanía digital & $\begin{array}{l}\text { Procurar su identidad digital y cuidar su información } \\
\text { personal }\end{array}$ \\
\hline Saber crear y manipular medios y multimedia & Tomar fotografías con un teléfono inteligente \\
\hline
\end{tabular}

Cada uno de los 10 capítulos del MOOC requiere de cuatro horas de dedicación por parte de los participantes. Los capítulos han sido diseñados para que docentes de educación básica, principalmente, incorporen a sus prácticas académicas herramientas y soluciones tecnológicas.

El MOOC está pensado para que docentes trasciendan el uso genérico de las TIC y las incorporen más allá del procesador de palabras y el administrador de presentaciones a sus tareas de planeación y docencia; se asume un nivel de compromiso por parte de los participantes que se retribuye con un nivel de confianza para la realización de tareas. Algunas de las actividades del MOOC hacen uso de un botón que debe ser presionado cuando el participante concluye y se siente satisfecho con el resultado. Este botón representa una prueba de confianza de los diseñadores y de compromiso por parte de los participantes.

Los componentes del MOOC son: introducción y aprendizajes esperados; presentación de contenidos; descripción del proyecto del capítulo; serie de tareas; y módulo de revisión de avances. Cada capítulo tiene etapas en las que se introduce el tema, se movilizan los saberes digitales de los participantes y se facilita la verificación de su aprendizaje.

Cada capítulo incluye: 1 . Tareas evaluables y cuya realización correcta proporciona puntos y 2. Un componente práctico en la forma de un proyecto informático que deberá desarrollarse a partir de la resolución de tareas específicas.

Los capítulos se acreditan obteniendo, al menos, el 70\% de la puntuación.

En total, el presente MOOC tiene 84 puntos posibles distribuidos de la siguiente forma (ver Tabla 2): 
Tabla 2. Puntos por capítulo

\begin{tabular}{lllllllllll}
\hline Capítulo & 1 & 2 & 3 & 4 & 5 & 6 & 7 & 8 & 9 & 10 \\
\hline $\begin{array}{l}\text { Número de } \\
\text { actividades (puntaje) }\end{array}$ & 9 & 9 & 7 & 9 & 9 & 11 & 7 & 7 & 6 & 10 \\
\hline
\end{tabular}

\section{METOdOLOGíA}

La primera emisión del curso, que es la que aquí se analiza, tuvo una duración de cinco semanas entre el mes de octubre y noviembre de 2018. A este curso se registraron 8,142 personas. La base de datos con la información de los inscritos al MOOC fue proporcionada por la plataforma México X, respetando en todo momento la confidencialidad de los participantes. El análisis es de enfoque cuantitativo y tiene un alcance descriptivo. Se depuró y limpió la base de datos y posteriormente se calcularon las frecuencias de las variables poblacionales (edad, sexo y nivel educativo). Posteriormente, se hicieron conteos de la participación en cada sección. Finalmente, con el fin de sintetizar los datos, se crearon tres grupos homogéneos o clusters usando la técnica de conglomerados jerárquicos (Johnson y Wichern, 2002).

El presente trabajo busca responder a las siguientes preguntas de investigación:

1. ¿Es un MOOC un recurso útil para capacitar docentes en activo?

2. ¿Existen diferencias en el desempeño de los participantes de los distintos perfiles demográficos?

3. ¿Existe una relación entre el perfil demográfico y la aprobación del curso?

4. ¿Existe un momento en el curso en el que los participantes optan por desertar?

\section{ANÁLISIS Y RESULTADOS}

Con base en información colectada con los usuarios de la plataforma México X se presentan resultados de investigación sobre la participación y el logro de los 8142 participantes en el MOOC Saberes Digitales para Docentes Nivel 1. Se buscó reconocer diferencias en el desempeño y hacer avanzar el conocimiento sobre la pertinencia de esta modalidad de estudios para la capacitación de docentes en activo.

\subsection{Características de la población de estudios}

\subsubsection{Distribución etaria}

Los participantes del MOOC se distribuyeron de la siguiente manera: del total de 8,142 participantes $570(7 \%)$ corresponden a personas que llamaremos Mayores, que nacieron entre los años 1946 y 1964 y que actualmente tienen más de 54 años; 2,742 (33\%) son personas a los que denominaremos Adultos pues nacieron entre los años 1965 y 1980 y tienen entre 39 y 54 años; $3,742(45 \%)$ los nombramos Jóvenes dado que nacieron entre 1981 y 1996 y tienen entre 23 y 38 años, y finalmente; 499 (6\%) son personas Muy jóvenes nacidas de 1997 en adelante y tienen menos de 23 años.

Como podrá observarse (Tabla 3) fueron las poblaciones Jóvenes y Adultos quienes tuvieron mayor participación en el curso. Según la experiencia internacional, las personas entre los 25 y 34 los de edad representa el $40 \%$ de los participantes en los cursos MOOC (Cole y Timmerman, 2015), cifra equivalente a la observada en este estudio en la población con edades entre 23 y 38 años (45\%). No obstante, es interesante saber que la población del grupo Adultos participó con el 33\% y que 570 personas mayores de 54 años se inscribieron a este curso. Esto se explica, en parte, por el hecho de que la población objetivo era los profesores en servicio de México que tienen, en promedio, 42.4 años de edad (IMCO, 2014). 
Tabla 3. Distribución etaria de la población

\begin{tabular}{lcc}
\hline Grupos de edad & Frecuencia & Porcentaje \\
\hline Mayores (más de 54 años) & 570 & 7.00 \\
Adultos (de 39 a 54 años) & 2,742 & 33.68 \\
Jóvenes (de 23 a 38 años) & 3,742 & 45.96 \\
Muy jóvenes (menos de 23 años) & 489 & 6.01 \\
Sin datos & 599 & 7.35 \\
Total & 8,142 & 100.00 \\
\hline
\end{tabular}

\subsubsection{Distribución por sexo}

En cuanto a distribución por sexo (Tabla 4), es importante destacar la participación de las mujeres en este curso. Casi el $56 \%$ de los participantes fueron mujeres, frente a $37 \%$ de hombres; si no tomamos en cuenta los participantes Sin datos, los porcentajes serían $60 \%$ y $40 \%$ respectivamente, casi la misma diferencia porcentual, $20 \%$, a favor de las mujeres. En la investigación reportada sobre estos cursos suele ser mayor la participación de los hombres (Dillahunt, Wang, y Teasley, 2014; R. F. Kizilcec, Piech, y Schneider, 2013); sin embargo, al ser un MOOC dirigido a docentes, es explicable esta mayor participación femenina. Además de que en México el profesorado de educación básica se integra mayoritariamente por mujeres con $59.6 \%$ frente a $40.3 \%$ de hombres (IMCO, 2014).

Tabla 4. Distribución por sexo

\begin{tabular}{lcc}
\hline Sexo & Frecuencia & Porcentaje \\
\hline Mujer & 4,532 & 55.66 \\
Hombre & 3,015 & 37.03 \\
Sin definir & 595 & 7.31 \\
Total & 8,142 & 100.00 \\
\hline
\end{tabular}

\subsubsection{Distribución por nivel educativo}

En este curso casi el $50 \%$ de quienes participaron reportó tener estudios de licenciatura, $23 \%$ de maestría, $10 \%$ bachillerato, y casi $5 \%$ doctorado (Tabla 5). Estos cursos son tomados mayoritariamente por personas que poseen estudios superiores (Bates, 2014; Ho et al., 2014), más aún en el caso de un curso dirigido a los docentes en activo.

Tabla 5. Distribución por nivel educativo

\begin{tabular}{lcc}
\hline Nivel de Educación & Frecuencia & Porcentaje \\
\hline Básica & 166 & 2.04 \\
Preparatoria & 844 & 10.37 \\
Licenciatura & 3,853 & 47.32 \\
Diplomado & 356 & 4.37 \\
Maestría & 1,891 & 23.23 \\
Doctorado & 375 & 4.61 \\
Educación informal & 5 & 0.06 \\
Otro tipo de educación & 69 & 0.85 \\
Sin definir & 583 & 7.15 \\
Total & 8,142 & 100.00 \\
\hline
\end{tabular}




\subsection{Resultados obtenidos por los participantes}

En primer lugar, se exponen los resultados sobre la participación y el desempeño global de los participantes con objeto de ponderar la eficacia general del curso y observar algunos de los desempeños que ponen en evidencia las propias actividades. En segundo lugar, se analiza el desempeño según los grupos de edad construidos. Y, en tercer lugar, y con el propósito de obtener una imagen de conjunto, se construyeron grupos o conglomerados que permitieran observar a mayor detalle el desempeño de los participantes.

\subsubsection{Participación y desempeño global}

A continuación, se muestra información sobre la participación y desempeño de los participantes en el MOOC. En la Tabla 6 se muestran los capítulos del curso en orden cronológico; se muestra el número de participantes, los puntajes medio obtenidos así como la desviación estándar. En todos los capítulos se observaron casos extremos de personas que obtenían 0 y otras que obtenían 1 que eran las calificaciones mínima y máxima.

Como podrá observarse, los mayores puntajes corresponden a Saber socializar y colaborar en entornos digitales y Saber ejercer y respetar una ciudadanía digital, seguidos de Saber crear y manipular medios y contenido multimedia. Los saberes con los menores puntajes corresponden a Literacidad digital, Saber administrar dispositivos y Saber administrar archivos. Fue notoria la dificultad que representó para los participantes realizar búsquedas de información en Internet usando operadores booleanos.

En la misma Tabla 6 y en laFigura 1 se puede observar la evolución del número de participantes por capítulo. Lo primero que se observa es una fuerte caída en la participación hasta el quinto capítulo a partir de cual el número de participante que abandonan el MOOC se ralentiza: de los 8,142 inscritos solamente 3,182 hicieron la primer actividad (39.08\%). Entre la primera y segunda actividad la participación disminuyó casi el 19\%; entre la segunda y la tercera actividad la participación disminuyó casi el 15\% en cada etapa; de la tercera a la quinta abandonaron otro 15\%. A partir de la quinta actividad la participación se mantuvo relativamente estable. Estas tendencias son similares a la información consignada por Clow (2013).

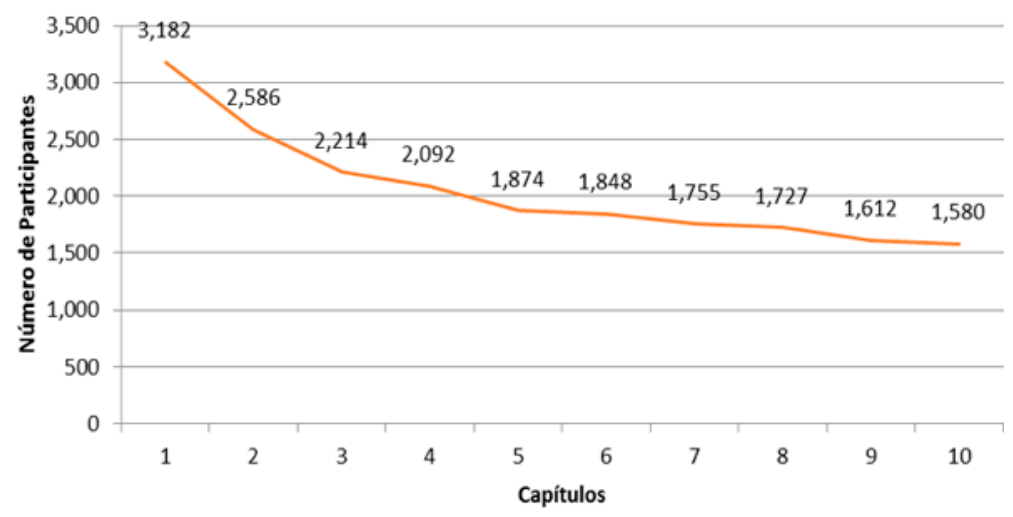

Figura 1. Participación por capítulo del curso

La aprobación del curso se otorga con el 70\% de las actividades realizadas adecuadamente, es decir al llegar al capítulo 7. Si se mide la aprobación contabilizando a todos los que se inscribieron, como se calcula normalmente, encontramos que poco más de la quinta parte de los participantes aprobaron el curso (ver Tabla 7), cifra que se asemeja a lo que ocurre internacionalmente (Onah, Sinclair, y Boyatt, 2014); pero si se mide contra los participantes que terminaron el primer capítulo resulta que el porcentaje sube a poco más del $53 \%$. Este último porcentaje de aprobación puede 
Tabla 6. Resultados de participación por capítulo del curso

\begin{tabular}{lcccc}
\hline Capítulos & $\begin{array}{c}\text { Número } \\
\text { de } \\
\text { participantes }\end{array}$ & $\begin{array}{c}\text { Variación } \\
\text { porcentual } \\
\text { de la } \\
\text { participación }\end{array}$ & $\begin{array}{c}\text { Calificación: } \\
\text { Media }\end{array}$ & $\begin{array}{c}\text { Calificación: } \\
\text { Desviación estándar }\end{array}$ \\
\hline $\begin{array}{l}\text { 1. Saber administrar } \\
\text { dispositivos }\end{array}$ & 3,182 & 100.00 & 0.82 & 0.26 \\
$\begin{array}{l}\text { 2. Saber administrar } \\
\text { archivos }\end{array}$ & 2,586 & -18.73 & 0.88 & 0.22 \\
$\begin{array}{l}\text { 3. Saber usar programas y } \\
\text { sistemas de información }\end{array}$ & 2,214 & -14.39 & 0.91 & 0.17 \\
especializados & & & & \\
$\begin{array}{l}\text { 4. Literacidad Digital } \\
\begin{array}{l}\text { 5. Saber crear y manipular } \\
\text { texto y texto enriquecido }\end{array}\end{array}$ & 1,874 & -10.42 & 0.94 & 0.18 \\
$\begin{array}{l}\text { 6. Saber crear y manipular } \\
\text { conjuntos de datos }\end{array}$ & 1,848 & -1.39 & 0.94 & 0.14 \\
$\begin{array}{l}\text { 7. Saber comunicarse en } \\
\text { entornos digitales }\end{array}$ & 1,755 & -5.03 & 0.94 & 0.13 \\
$\begin{array}{l}\text { 8. Saber socializar y } \\
\text { colaborar en entornos } \\
\text { digitales }\end{array}$ & 1,727 & -1.60 & 0.96 & 0.12 \\
$\begin{array}{l}\text { 9. Saber ejercer y respetar } \\
\text { una ciudadanía digital }\end{array}$ & 1,612 & -6.66 & 0.96 & 0.12 \\
$\begin{array}{l}\text { 10. Saber crear y manipular } \\
\text { medios y contenido } \\
\text { multimedia }\end{array}$ & 1,580 & -5.51 & 0.95 & \\
\hline
\end{tabular}

Tabla 7. Aprobación

\begin{tabular}{lcccc}
\hline \multirow{2}{*}{ Aprobado } & \multicolumn{2}{c}{ Sólo los que terminaron el primer } & \multicolumn{2}{c}{ Total de inscritos } \\
& capítulo & & Frecuencia & $\%$ \\
\hline No & 5,849 & 46.76 & 5,849 & 71.84 \\
Sí & 1,694 & 53.24 & 1,694 & 20.81 \\
Total & 3,182 & 100.00 & 8,142 & 100.00 \\
\hline
\end{tabular}

explicarse debido a que el curso fue dirigido a una población en particular que se inscribió voluntariamente con deseos de aprender.

\subsection{Las diferencias etarias}

En cuanto a la aprobación del curso, en la Tabla 8 se muestra que los participantes Mayores obtuvieron el mayor porcentaje de aprobación con 29\%, seguido de los Adultos con $25 \%$, después los Jóvenes con $20 \%$ y, por último, los Muy Jóvenes. Es interesante reconocer que fueron las personas de mayor edad quienes aprobaron mayormente el curso, dato que confirma lo reportado por Morris, Hotchkiss, y Swinnerton (2015). Este dato da cuenta de la participación creciente de dicho grupo etario en el uso funcional de las TIC y es evidencia de una motivación fortalecida para concluir el curso satisfactoriamente.

La participación en los foros fue una de las actividades distintivas por grupos de edad. La Tabla 9 muestra que los participantes Mayores, del grupo que nació entre 1946 y 1964, fueron quienes tuvieron el mayor porcentaje de participación en los foros con $22 \%$, seguido de los Adultos con $17 \%$, luego los Jóvenes con $11 \%$ y, al final, 
Tabla 8. Distribución de la aprobación por grupo etario

\begin{tabular}{lccccc}
\hline Aprobado & Mayores & Adultos & Jóvenes & Muy jóvenes & Total \\
\hline \multirow{2}{*}{ No } & 404 & 2,042 & 2,976 & 427 & 5,849 \\
& $70.87 \%$ & $74.47 \%$ & $79.52 \%$ & $87.32 \%$ & $77.54 \%$ \\
Sí & 166 & 700 & 766 & 62 & 1,694 \\
& $29.12 \%$ & $25.52 \%$ & $20.47 \%$ & $12.67 \%$ & $22.45 \%$ \\
Total & 570 & 2,742 & 3,742 & 489 & 7,543 \\
& $100.00 \%$ & $100.00 \%$ & $100.00 \%$ & $100.00 \%$ & $100.00 \%$ \\
\hline
\end{tabular}

los Muy jóvenes con 4\%. De nueva cuenta la población de mayor edad participa más en los espacios de colaboración disponibles en el curso, dato que refuerza lo reportado por Huang, Dasgupta, Ghosh, Manning, y Sanders (2014). Se sabe que la colaboración en los espacios virtuales favorece el intercambio de puntos de vista y permite la construcción conjunta de significados (Bates, 2014); por ello, la participación en los foros de discusión contribuye a la construcción colectiva del conocimiento.

Tabla 9. Participación en foros por grupo etario

\begin{tabular}{lccccc}
\hline $\begin{array}{l}\text { Participación } \\
\text { en Foros }\end{array}$ & Mayores & Adultos & Jóvenes & Muy jóvenes & Total \\
\hline No & 443 & 2,265 & 3,309 & 466 & 6,483 \\
& $77.71 \%$ & $82.60 \%$ & 88.42 & 95.29 & $85.94 \%$ \\
Sí & 127 & 477 & 433 & 23 & 1,060 \\
& $22.28 \%$ & $17.39 \%$ & 11.57 & 4.70 & $14.05 \%$ \\
Total & 570 & 2,742 & 3,742 & 489 & 7,543 \\
& $100.00 \%$ & $100.00 \%$ & $100.00 \%$ & $100.00 \%$ & $100.00 \%$ \\
\hline
\end{tabular}

\subsection{Una visión de conjunto}

Con el fin de conocer a mayor detalle el desempeño de los participantes, en este apartado se analiza el caso solamente de quienes terminaron el curso. Se trata de una población de 1,559 personas. Se construyeron tres grupos a través de la técnica de clusters (Johnson y Wichern, 2002). Se llevó a cabo un análisis de conglomerados, que separó a tres grupos cuyo rendimiento en el curso fue notablemente diferenciado. La Figura 2 muestra el desempeño de los tres grupos en cada uno de las diez tipos de capítulos o módulos del curso.

Como puede observarse, los participantes del cluster 3, el de máximo desempeño, obtuvo un puntaje de casi 1 en los diez módulos, salvo el 4, correspondiente a Literacidad digital, que, como se dijo antes, representó la mayor dificultad para todos los participantes. El cluster 2, de buen desempeño, obtuvo puntajes que oscilaron entre 0.9 y 1.0, salvo en la actividad 4 cuyo puntaje bajó a 0.7 . El cluster 1, con desempeño regular, obtuvo puntajes entre 0.65 y 0.8 , mismos que representaron los menores de toda la población.

La Tabla 10 nos permite ver que del total de quienes terminaron el curso la enorme mayoría tuvo un desempeño muy satisfactorio ya que únicamente el $7.44 \%$ tuvieron un desempeño regular.

Destaca que la actividad 4 de Literacidad digital representó una mayor dificultad para los grupos con desempeño bueno y regular y no tanto para el de máximo desempeño.

La Tabla 11 muestra la distribución etaria de los tres clusters. Como puede observarse fueron los Jóvenes quienes representaron en mayor porcentaje al grupo de Máximo 


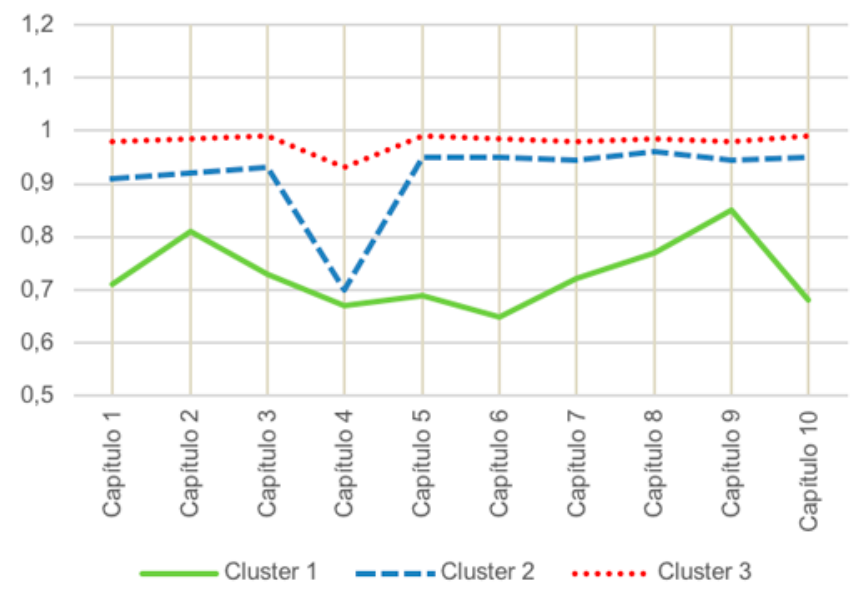

Figura 2. Análisis de conglomerados

Tabla 10. Distribución de la población en tres grupos en función de su rendimiento

\begin{tabular}{lcc}
\hline Cluster & Frecuencia & $\%$ \\
\hline 1. Desempeño regular & 116 & 7.44 \\
2. Buen desempeño & 542 & 34.77 \\
3. Máximo desempeño & 901 & 57.79 \\
Total & 1,559 & 100.00 \\
\hline
\end{tabular}

desempeño con 61\%; seguidos de los Mayores con 57\%; luego los Adultos con 54\%; y, por último, los Muy jóvenes con 50\%. Este grupo etario fue el que contribuyó en mayor medida a la integración del grupo con desempeño regular con $14 \%$, mientras que fueron los Mayores quienes lo representaron en menor medida con $4 \%$.

Tabla 11. Distribución de grupos etarios en clusters de desempeño

\begin{tabular}{|c|c|c|c|c|c|c|c|c|}
\hline \multicolumn{3}{|c|}{$\begin{array}{c}\text { Cluster } 1 \\
\text { Grupos etarios Desempeño } \\
\text { regular }\end{array}$} & \multicolumn{2}{|c|}{$\begin{array}{l}\text { Cluster } 2 \\
\text { Buen desempeño }\end{array}$} & \multicolumn{2}{|c|}{$\begin{array}{l}\text { Cluster } 3 \\
\text { Máximo desempeño }\end{array}$} & \multicolumn{2}{|c|}{ Total } \\
\hline & Frecuencia & $\%$ & Frecuencia & $\%$ & Frecuencia & $\%$ & Frecuencia & $\%$ \\
\hline Mayores & 7 & 4.26 & 63 & 38.42 & 94 & 57.32 & 164 & 100.00 \\
\hline Adultos & 47 & 7.35 & 243 & 37.97 & 350 & 54.68 & 640 & 100.00 \\
\hline Jóvenes & 52 & 7.69 & 211 & 31.22 & 413 & 61.09 & 676 & 100.00 \\
\hline $\begin{array}{l}\text { Muy } \\
\text { jóvenes }\end{array}$ & 9 & 14.76 & 21 & 34.43 & 31 & 50.81 & 61 & 100.00 \\
\hline $\begin{array}{l}\text { Sin } \\
\text { datos }\end{array}$ & 1 & 5.56 & 4 & 22.22 & 13 & 72.22 & 18 & 100.00 \\
\hline Total & 116 & 7.45 & 542 & 34.76 & 901 & 57.79 & 1,559 & 100.00 \\
\hline
\end{tabular}

En la Tabla 12 se muestra la distribución de los integrantes de los tres grupos por nivel educativo. El grupo que reportó estudios de Doctorado fue el que mayor representación tuvo en el grupo con máximo desempeño con $61 \%$, seguido de quienes reportaron tener una Licenciatura con 59\%, luego el nivel de Bachillerato con 56\%. Los niveles educativos que integraron al tercer grupo en mayor medida fueron quienes reportaron tener un Diplomado con 15\% y Preparatoria con $12 \%$.

En la Tabla 12 se puede observar que hay una correspondencia entre estudios superiores y desempeño en el MOOC favoreciendo a quienes detentan las credenciales académicas más altas. 
Tabla 12. Distribución de integrantes de grupos por nivel educativo

\begin{tabular}{lcccccccc}
\hline \multicolumn{3}{c}{ Nivel Educativo } & \multicolumn{7}{c}{ Desempeño regular } & \multicolumn{2}{c}{ Buen desempeño } & \multicolumn{2}{c}{ Máximo desempeño } \\
Frecuencia & $\%$ & Frecuencia & $\%$ & Frecuencia & $\%$ & Frecuencia & $\%$ \\
\hline Básica & 0 & 0.00 & 1 & 100.00 & 0 & 0.00 & 1 & 100.00 \\
Secundaria & 1 & 10.00 & 7 & 70.00 & 2 & 20.00 & 10 & 100.00 \\
Preparatoria & 15 & 12.94 & 35 & 30.17 & 66 & 56.89 & 116 & 100.00 \\
Licenciatura & 55 & 7.19 & 255 & 33.34 & 455 & 59.47 & 765 & 100.00 \\
Maestría & 29 & 6.15 & 173 & 36.65 & 270 & 57.20 & 472 & 100.00 \\
Doctorado & 5 & 4.68 & 36 & 33.64 & 66 & 61.68 & 107 & 100.00 \\
Diplomado & 10 & 15.88 & 27 & 42.86 & 26 & 41.26 & 63 & 100.00 \\
Otro tipo & 1 & 9.09 & 3 & 27.27 & 7 & 63.64 & 11 & 100.00 \\
Sin & 0 & 0.00 & 5 & 35.71 & 9 & 64.29 & 14 & 100.00 \\
datos & & & & & & & & \\
Total & 116 & 7.45 & 542 & 34.76 & 901 & 57.79 & 1,559 & 100.00 \\
\hline
\end{tabular}

En cuanto al género, no se encontraron diferencias significativas en los clusters como puede verse en la Tabla 13.

Tabla 13. Distribución de integrantes de grupos por sexo

\begin{tabular}{lcccccccc}
\hline Sexo & \multicolumn{2}{c}{ Cluster 1 } & \multicolumn{2}{c}{ Cluster 2 } & \multicolumn{2}{c}{ Cluster 3 } & \multicolumn{2}{c}{ Total } \\
& \multicolumn{2}{c}{ Desempeño regular } & \multicolumn{2}{c}{ Buen desempeño } & \multicolumn{2}{c}{ Máximo desempeño } & & \\
& Frecuencia & $\%$ & Frecuencia & $\%$ & Frecuencia & $\%$ & Frecuencia & $\%$ \\
\hline Mujer & 59 & 6.67 & 326 & 36.83 & 500 & 56.50 & 885 & 100.00 \\
Hombre & 57 & 8.62 & 211 & 31.92 & 393 & 59.45 & 661 & 100.00 \\
Sin & 0 & 0.00 & 5 & 0.18 & 8 & 0.00 & 13 & 100.00 \\
datos & & & & & & & & \\
Total & & & 542 & 34.76 & 901 & 57.79 & 1,559 & 100.00 \\
\hline
\end{tabular}

\section{CONCLUSIONES}

Este estudio es un primer acercamiento en México para conocer los patrones de participación y logro de un MOOC dirigido a la capacitación de docentes en servicio. Los resultados obtenidos de la participación y logro de los participantes en el MOOC Saberes Digitales para Docentes Nivel 1 indican que la formación de docentes en servicio puede realizarse mediante una oferta educativa en esta modalidad. En esta experiencia más de $90 \%$ de la población que concluyó el MOOC obtuvo una evaluación de bueno o máximo desempeño. Sin desconocer que la eficiencia terminal de $23 \%$ no fue favorable, en términos absolutos, fueron más de 1530 personas que vivieron una experiencia positiva de aprendizaje y mostraron un alto desempeño en el curso. Es más, si se considera ese indicador a partir de quienes realizaron la primera actividad el porcentaje sube a 53\%. Quienes tienen interés y poseen capacidad autogestiva son quienes más se benefician de esta modalidad (R. Kizilcec y Halawa, 2015; Margaryan, Littlejohn, y Milligan, 2013).

Los resultados obtenidos en este estudio indican desempeños diferenciados entre las distintas distribuciones etarias y nivel de estudios. Por una parte, fue la población de mayor edad quienes aprobaron mayoritariamente el MOOC y obtuvieron mejores tasas de aprovechamiento de los recursos de interacción. Esta información confirma los resultados obtenidos por Morris et al. (2015) quienes encontraron que los parti- 
cipantes que concluyeron más del $50 \%$ de un MOOC fueron quienes tenían mayor edad (mediana de 42.7 años) y quienes optaron por separarse del curso en la primera semana fue la población de menor edad (mediana de 34.3 años). No obstante, en este estudio fueron los Jóvenes quienes obtuvieron el mayor porcentaje en el cluster de Máximo Rendimiento. Como se reporta en la literatura (Liyanagunawardena, Adams, y Williams, 2013; Rohs y Ganz, 2015) quienes poseen un mayor nivel educativo participan y obtienen mejores desempeños en los cursos MOOC. En esta experiencia quienes reportaron poseer el nivel de Doctorado fueron quienes representaron el mayor porcentaje en el cluster de máximo rendimiento. En cuanto a género no se observaron diferencias significativas en los tres niveles de rendimiento, si bien los hombres superaron ligeramente a las mujeres en la categoría de máximo rendimiento.

La participación global adoptó la forma de embudo confirmando lo reportado por Clow (2013) y Jordan (2015) en donde se observa una disminución aguda de la participación a partir de la primera actividad y estabilizándose hacia la quinta. Este resultado también es consistente con lo reportado por (Glance y Barrett, 2014) que encontraron, después de revisar los patrones de participación y las tasas de eficiencia terminal de los participantes en 42 cursos ofrecidos por la Universidad de Stanford, que existe un descenso notable de la participación a partir de la primera semana de actividad para después mantener la tasa de participación relativamente estable.

Por la información obtenida y los análisis llevados a cabo en este estudio es posible responder las preguntas formuladas de investigación y afirmar que los MOOC representan una estrategia eficaz de formación de docentes de educación básica en servicio. Asimismo, el estudio hace evidente que existe un desempeño diferenciado y de logro en función de características demográficas y, por último, que la participación decrece significativamente después del inicio del curso y se estabiliza a partir de la quinta actividad.

Las necesidades de formación permanente de los maestros mexicanos en servicio para lograr la apropiación de los saberes digitales pueden satisfacerse mediante una estrategia nacional que promueva el uso de MOOCs, como en el caso de Uganda Oyo, Kalema, y Byabazaire (2017). Los maestros en servicio responden positivamente a estas modalidades de capacitación continua como lo constata la población participante en este curso.

El diseño, gestión y facilitación del MOOC de saberes digitales para docentes, dejó enseñanzas de forma y fondo al equipo de trabajo. Se observó que el capítulo de Literacidad Digital fue el más complicado para esta cohorte y no solo por la temática sino también por el tipo de actividades que se solicitaron realizar. El ejercicio más conflictivo solicitaba que el participante escribiera en un campo una serie de palabras para realizar una búsqueda avanzada en Internet. Sin embargo, la sintaxis de las respuestas esperadas, las diferencias en el manejo de mayúsculas, minúsculas, omisión de acentos y otras variantes léxicas complicaron la identificación de las respuestas correctas, por lo que para otras emisiones del curso el campo abierto se cambió por opción múltiple.

El curso descansó sobre una base de confianza en la que se asume al participante como un par académico que ha optado por tomar voluntariamente el curso con el propósito principal de aprender. Por lo que desmontar la fiscalización de recursos como método de evaluación y considerar la auto percepción del desempeño como guía de avance es consistente con la filosofía de este tipo de cursos. En este sentido, el MOOC de Saberes Digitales para Docentes Nivel 1 representa una innovación en el esquema de evaluación empleado, al darle crédito a la palabra del participante y considerar la apreciación de su desempeño como parte de los elementos considerados en la calificación por módulo y final. Sin duda, los MOOC ofrecen amplias oportunidades para innovar medios de evaluación que sirvan para reconocer la responsabilidad y compromiso de superación de los maestros en activo. 
Otro tema que deberá continuar analizándose a futuro es el relativo a cómo evaluar la eficiencia y eficacia de los MOOC (Onah et al., 2014) ya que son muchas las razones por las cuales una persona se inscribe a estos cursos que puede no necesariamente ser el seguir el curso en su totalidad. En este estudio fue evidente que quienes realizaron la primera actividad fueron los que mayoritariamente concluyeron el curso. Hay quienes sólo se registraron para conocer la plataforma y el contenido del curso, pero sin llevar a cabo alguna de las actividades y si se mide la eficiencia terminal con esta población siempre será menor a si se valora con base en quienes realizaron al menos una actividad.

Los MOOC poseen un enorme potencial para contribuir a satisfacer necesidades educativas de múltiples temas y poblaciones. Es deseable que México construya una estrategia que promueva la oferta de cursos MOOC para la capacitación de los maestros en servicio y en otras áreas profesionales. El favorable crecimiento observado en la oferta de MOOCs en México deberá acompañarse de estudios sistemáticos que arrojen información sobre los participantes, sus trayectorias y los aprendizajes logrados.

\section{REFERENCIAS}

Bates, T. (2014, 19 de 10). Strengths and weaknesses of MOOCs. Part I. [Blog post]. Descargado de

https://www.tonybates.ca/2014/10/19/the-strengths-and-weaknesses-of-moocs-part-i/

Baturay, M. H. (2015). An Overview of the World of MOOCs. Procedia-Social and Behavioral Science, 174, 427-433. https://doi.org/10.1016/j.sbspro.2015.01.685

Cassany, D., y Lenguaje y Textos. (2014). Cinco buenas prácticas de enseñanza con internet. , 39, 39-47. Descargado de http://hdl.handle.net/10230/27136

Clow, D. (2013). MOOCs and the funnel of participation. Third Conference on Learning Analytics and Knowledge (LAK 2013) (pp. 185-189). Descargado de https://oro.open.ac.uk/36657/1/DougClow-LAK13-revised-submitted.pdf

Coffrin, C., Corrin, L., de Barba, P., y Kennedy, G. (2014). Visualizing patterns of student engagement and performance in MOOCs. LAK'14 Proceedings of the Fourth International Conference on Learning Analytics and Knowledge (pp. 83-92). https://doi.org/10.1145/2567574.2567586

Cole, A. W., y Timmerman, C. E. (2015). What do current college students think about MOOCs? MERLOT Journal of Online Learning and Teaching, 11(2), 188-201. Descargado de https://jolt.merlot.org/Vol11no2/Cole_0615.pdf

Dellarocas, C., y Alstybne, M. V. (2013). Money Models For MOOCs. Communications of the $A C M, 56,25-28$. Descargado de

https://cacm.acm.org/magazines/2013/8/166304-money-models-for-moocs/fulltext

Dikke, D., y Faltin, N. (2015). Go_Lab MOOC- an online course for teacher professional development in the field of inquiry based science education. 7th International Conference on Education and New Learning Technologies (pp. 1206503-1206503). Descargado de https://telearn.archives-ouvertes.fr/hal-01206503/document

Dillahunt, T., Wang, B. Z., y Teasley, S. D. (2014). Democratizing higher education: Exploring MOOC use among those who cannot afford a formal education. International Review of Research in Open and Distance Learning, 15(5), 177-196.

https://doi.org/10.19173/irrodl.v15i5.1841

Domínguez, J., y Morcillo, S. (2016). Evaluación de un curso en línea para la formación de competencias en el uso de las TIC en profesores de ciencias de secundarias públicas del sureste de México. Revista de Educación a Distancia. Revista de Educación a Distancia, 51, 1-25. http://dx.doi.org/10.6018/red/51/2

Downes, S. (2012, 23 de 04). The Rise of MOOCs. [Blog post]. Descargado de https://www.downes.ca/cgi-bin/page.cgi?post=57911 
Drake, J. R., Hara, M. O., y Seeman, E. (2015). Five Principles of MOOC Design: With a Case Study. Journal of Information Technology Education: Innovations in Practice, 14, 125-143. Descargado de http://www.jite.org/documents/Vol14/JITEv14IIPp125-143Drake0888.pdf

Florentine, S. (2015, 28 de 09). 4 ways MOOCs are changing teacher development. Descargado de https://www.cio.com/article/2986306/ 4-ways-moocs-are-changing-professional-development.html

García, P., Ibarra, C., y Sandoval, K. (2018). Cursos MOOC: Una alternativa de formación docente en CONALEP. En R. Mercado-del Collado (Ed.), Cursos masivos abiertos en línea (MOOCs). El caso de México (Vol. 6, pp. 169-182). Argentina: Brujas /Social TIC.

Glance, D. G., y Barrett, P. H. R. (2014). Attrition patterns amongst participant groups in Massive Open Online Courses. Annual Conference of the Australian Society for Computers in Tertiary Education 2014 (Vol. 31, pp. 12-20). Australasian Society for Computers in Learning in Tertiary Education. Descargado de https://research-repository.uwa.edu.au/en/publications/ attrition-patterns-amongst-participant-groups-in-massive-open-onl

Gulbahar, Y., Kalelioglu, F., y Bahadir-Kert, S. (2018). Teaching Computational Thinking to In-Service Computer Science Teachers through a Massive Open. Proceedings of E-Learn: World Conference on E-Learning in Corporate, Government, Healthcare, and Higher Education (pp. 922-928). AACE. Descargado de https://www.learntechlib.org/primary/p/185051

Ho, A., Reich, J., Nesterko, S., Seaton, D., Mullaney, T., Waldo, J., y Chuang, I. (2014). HarvardX and MITx: The First Year of Open Online Courses, Fall 2012-Summer 2013. Descargado de https://papers.ssrn.com/sol3/papers.cfm?abstract_id=2381263

Huang, J., Dasgupta, A., Ghosh, A., Manning, J., y Sanders, M. (2014). Superposter behavior in MOOC forums. Proceedings of the first ACM conference on Learning \& Scale. http://dx.doi.org/10.1145/2556325.2566249

IMCO. (2014). Mapa del magisterio de educación básica de México. Descargado de https:// imco.org.mx/capital_humano/mapa-del-magisterio-de-educacion-basica-en-mexico/

Johnson, R. A., y Wichern, D. W. (2002). Applied Multivariate Statistical Analysis. London: Prentice Hall.

Jordan, K. (2015). Massive open online course completion rates revisited: Assessment, length and attrition. The International Review of Research in Open and Distributed Learning, 16(3), 341-358. https://doi.org/10.19173/irrodl.v16i3.2112

Kaul, M., Aksela, M., y Wu, X. (2018). Dynamics of the community of inquiry (Col) within a massive open online course (MOOC) for in-service teachers in environmental education. Dirasat. Educational Sciences, 8(2), 40-40.

https://doi.org/10.3390/educsci8020040

Kizilcec, R., y Halawa, S. (2015). Attrition and Achievement Gaps in Online Learning. Proceeding L@S '15 Proceedings of the Second (2015) ACM Conference on Learning. https://doi.org/10.1145/2724660.2724680

Kizilcec, R. F., Piech, C., y Schneider, E. (2013). Deconstructing Disengagement: Analyzing Learner Subpopulations in Massive Open Online Courses. Proceedings of the Third International Conference on Learning Analytics and Knowledge (Vol. 13, pp. 10-10).

Kumari, A. (2016). MOOCs - An online platform for teacher professional development. Asian Journal of Multidisciplinary Studies, 4(5), 102-107. Descargado de http://www.ajms.co.in/sites/ajms2015/index.php/ajms/article/view/1799/pdf_26

Laurillard, D. (2016). The educational problem that MOOCs could solve: professional development for teachers of disadvantage students. Research in Learning Technology, 24, 1-17. https://doi.org/10.3402/rlt.v24.29369

León-Urrutia, M., Fielding, S., y White, S. (2016). Professional Development Through MOOCs in Higher Education Institutions: Challenges and Opportunities for PhD Students Working as Mentors. Journal of Interactive Media in Education, 1, 1-10. 


\section{http://doi.org/10.5334/jime.427}

Liyanagunawardena, T. R., Adams, A. A., y Williams, S. A. (2013). MOOCs: A systematic study of the published literature 2008-2012. The International Review of Research in Open and Distance Learning, 14, 202-227. Descargado de http://www.irrodl.org/index.php/irrodl/article/view/1455/2531

Margaryan, A., Littlejohn, A., y Milligan, C. (2013). Self-regulated learning in the workplace: Strategies and factors in the attainment of learning goals. International fournal of Training and Development, 17(4), 245-259. https://doi.org/10.1111/ijtd.12013

Mercado-del Collado, R., Downes, S., Ramírez-Montoya, M., Farías-Gaytán, S., Morales-Rodríguez, A., Olguín-Aguilar, P., ... García-Zúñiga, M. G. (2018). Cursos masivos abiertos en línea MOOCs: El caso de México (Vol. 6; R. M. del Collado, Ed.). Argentina: Brujas / Social TIC. Descargado de https://www.uv.mx/blogs/brechadigital/files/2018/12/hdt6.pdf

Misra, P. K. (2018). MOOCs for Teacher Professional Development: Reflections and Suggested Actions. Open Praxis, 10(1), 67-77. http://dx.doi.org/10.5944/openpraxis.10.1.780

Morris, N., Hotchkiss, S., y Swinnerton, B. (2015). Can demographic information predict MOOC learner outcomes? . Experience Track: Proceedings of the European MOOC Stakeholder. Descargado de http://eprints.whiterose.ac.uk/86184/

Onah, D., Sinclair, J., y Boyatt, R. (2014). Dropout Rates of Massive Open Online Courses: Behavioural Patterns. 6th International Conference on Education and New Learning Technologies (EDULEARN14) (pp. 5825-5834). Descargado de http://wrap.warwick.ac.uk/65543/1/WRAP_9770711-cs-070115-edulearn2014.pdf

Oyo, B., Kalema, B., y Byabazaire, J. (2017). Los MOOC para profesores en ejercicio: El caso de Uganda y las lecciones para África. Revista Española de Pedagogía, 75(266), 121-141. https://doi.org/10.22550/REP75-1-2017-07

Patru, M., y Balaji, V. (2016). Making Sense of MOOCs: A guide for policy-makers in developing countries (M. Patru y V. Balaji, Eds.). UNESCO / Commonwealth of Learning.

Phan, T., McNeil, S., y Robin, B. (2016). Students' patterns of engagement and course performance in a Massive Online Course. Computers \& Education, 95, 36-44. https://doi.org/10.1016/j.compedu.2015.11.015

Ramírez-Martinell, A., y Casillas, M. (2018). MOOC: Saberes Digitales para Docentes. México: Lulu. Descargado de https://www.uv.mx/blogs/brechadigital/files/2018/12/MOOC-Saberes-Digitales.pdf

Ramírez-Martinell, A., y Casillas, M. A. (2015). Los saberes digitales de los universitarios. En J. Micheli et al. (Eds.), Educación virtual y universidad. Un modelo de evolución (Vol. 17, pp. 77-106). México: Universidad Autónoma Metropolitana.

Ramírez-Martinell, A., y Casillas, M. A. (2017). Saberes digitales de los docentes de educación básica. Una propuesta para la discusión desde Veracruz. Descargado de https:// www.uv.mx/personal/albramirez/files/2015/06/Saberes-Digitales-SEV-libro-final.pdf

Ramírez-Martinell, A., Casillas, M. A., y Aguirre, I. G. (2018). Habilitación tecnológica de profesores universitarios y docentes de educación básica. Apertura, 10(2), 129-139. http://dx.doi.org/10.18381/Ap.v10n2.1368

Ramírez-Montoya, M., y Farías-Gaytán, S. (2018). De los ambientes a distancia a los cursos masivos abiertos:el caso de los MOOC del Tec de Monterrey. En R. Mercado-del Collado (Ed.), Los MOOC: El caso México. Argentina: Editorial Brujas. Descargado de https://repositorio.tec.mx/bitstream/handle/11285/628943/ 180428-Cap\%20Mooc-Tec-UV.pdf?sequence $=1$

Rohs, M., y Ganz, M. (2015). MOOCs and the Claim of Education for All: A Disilussion by Emprirical Data. International Review of Research in Open and Distributed Learning, 16(6).

Shah, D. (2018, 11 de 12). By The Numbers: MOOCs in 2018. [Blog post]. Descargado de https://www.classcentral.com/report/mooc-stats-2018/

Silva-Peña, I., y Salgado, I. (2014). Use of MOOCs in Teacher Education: advantages, 
disadvantages and riks. Profesorado. Revista de Curriculum y Formación del Profesorado, 18(1), 155-166. Descargado de https://www.ugr.es/ recfpro/rev181ART9.pdf

Veracruzana), U. U. (2019). México X Saberes Digitales para Docentes Nivel 1. Descargado de https://www.mexicox.gob.mx/courses/course-v1:UV+SDPD19113X+2019_11/about

Zubieta, J., y Rama, C. (2015). La universidad a la vanguardia tecnológica: Los cursos masivos abiertos en línea (MOOC). La Educación a Distancia en México. Una nueva realidad universitaria. México: UNAM / Virtual Educa. Descargado de https://web.cuaed.unam.mx/wp-content/uploads/2015/09/PDF/educacionDistancia.pdf 